\title{
Undulator Beam Pipe Magnetic Shielding Effect Tests*
}

\author{
Andrew Fisher, Zachary Wolf \\ SLAC
}

July , 2007

Abstract

The proposed stainless steel beampipe for the LCLS undulator has a measureable shielding effect on the magnetic field of the LCLS undulators. This note describes the tests used to determine the magnitude of the shielding effect, as well as deviations in the shielding effect caused by placing different phase shims in the undulator gap. The effect of the proposed Steel strongback which will be used to support the beam pipe, was also studied.

\section{Introduction}

A hall probe on a 3 axis movement system was set up to measure the main component of the magnetic field in the Prototype Undulator. To account for for temperature variations of the magnetic field of the undulator for succesive tests, a correction is applied which is described in this technical note. Using this method, we found the shielding effect, the amount which the field inside the gap was reduced due to the placement of the beampipe, to be $\sim 10$ Gauss.

A series of tests was also performed to determine the effect of phase shims and $\mathrm{X}$ and $\mathrm{Y}$ correction shims on the shielding. The largest effect on shielding was found for the $.3 \mathrm{~mm}$ phase shims. The effect of the $.3 \mathrm{~mm}$ phase shims was to increase the shielding effect $\sim 4$ Gauss. The tolerance for the shielding effect of the phase shims is less than 1 gauss.

The effect of the strongback was seen in its permanent magnetic field. It introduced a dipole field across the measured secion of the undulator of $\sim 3$ gauss. This note documents the tests performed to determine these effects, as well as the results of those tests.

\section{Test Set Up}

\subsection{Setup Description}

The measurements were conducted using a Sypris Hall probe on a high precision Z movement stage. The stage had a movement precision of 1 micron and a linear scale which could measure the position to .2 microns. Two temperature probes were placed on the magnet surface to monitor temperature drift in the magnet, and a third to monitor ambient temperature. The procedure was to start at the end of the magnet and scan the hall probe along the $\mathrm{Z}$ axis, taking data every $1 \mathrm{~mm}$ with sufficient pause after each move to allow the field reading to stabilize. The step size was determined to give sufficient resolution to find the peaks of the fields, but minimize the time for each scan so as to decrease the effect of temperature variations in the magnet and hall probe. To help minimize the effect of temperature on the magnet, a correction was applied to the data of $.1 \% /{ }^{\circ} \mathrm{C}$ with $27^{\circ} \mathrm{C}$ used as the baseline temperature for correction. It was determined that the primary source of error in our measurements was due to the temperature drift of the hall probe. Although the hall probe itself is temperature compensated so that the offset drift would be less than .01 gauss $/{ }^{\circ} \mathrm{C}$, the drift in the gain of the probe at the fields we were measuring was closer to 2 gauss $/{ }^{\circ} \mathrm{C}$ according to the hall probe specifications. In order to determine the approximate size of the error, a procedure was developed to estimate the size of the error bars.

* Work supported in part by the DOE Contract DE-AC02-76SF00515. This work was performed in support of the LCLS project at SLAC. 


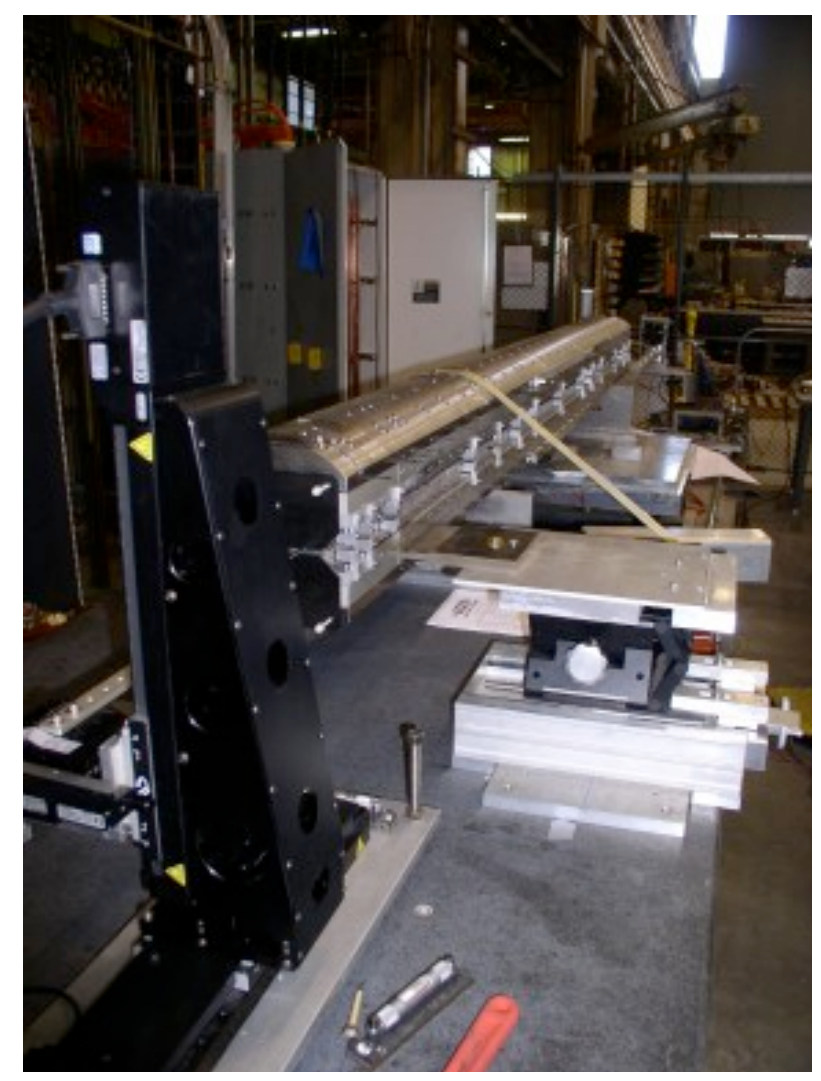

End on view of Undulator with Steel Beampipe.

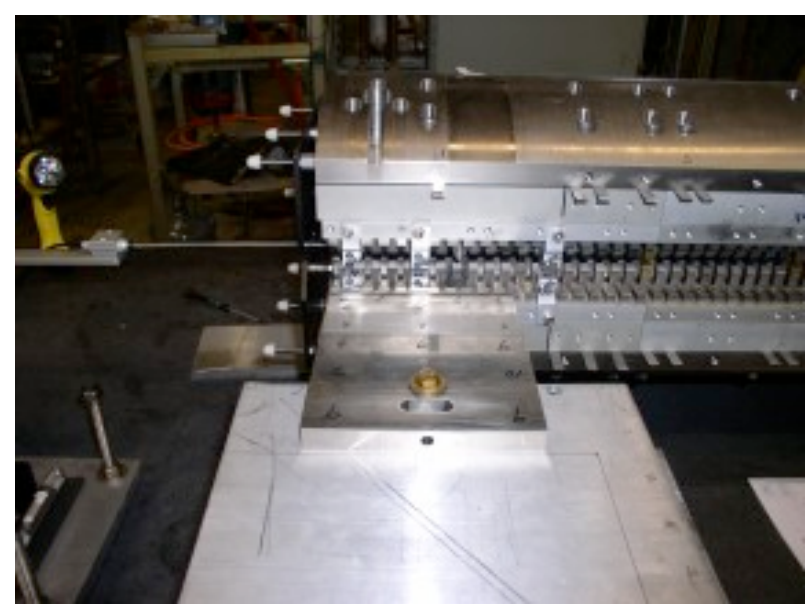

Side view of Undulator with Steel Beampipe. 


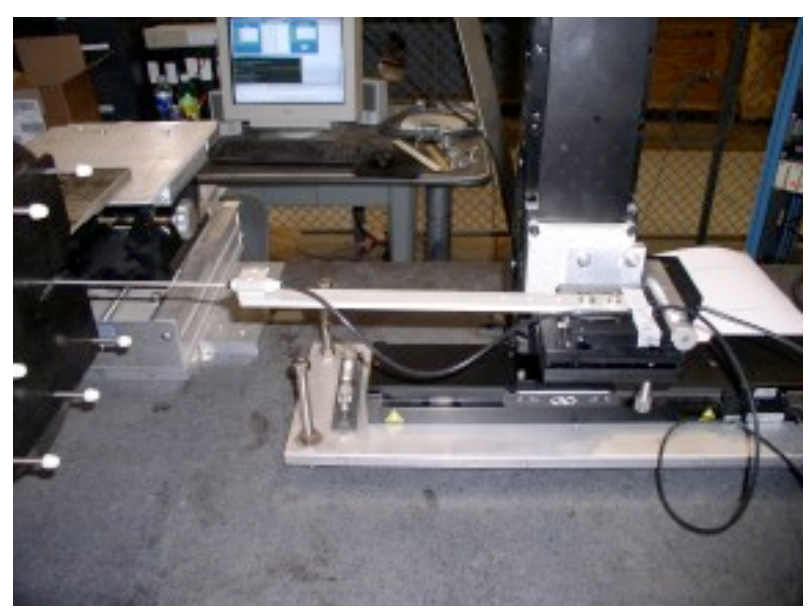

Hall Probe Setup.

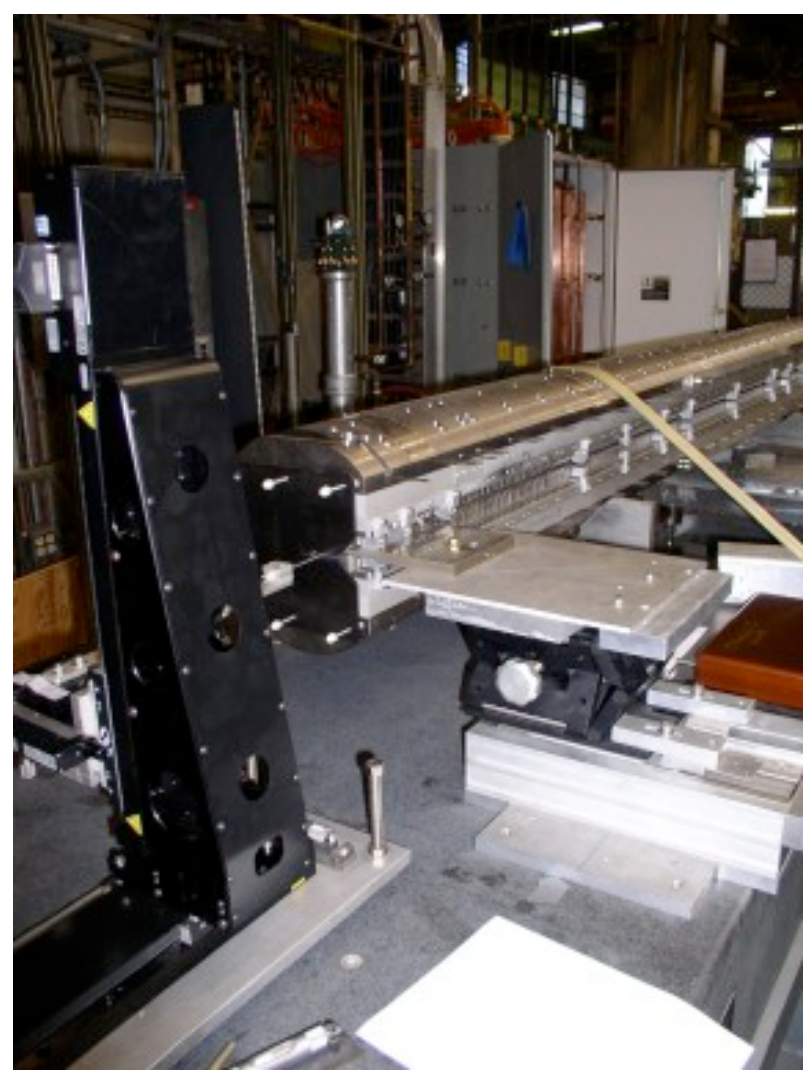

Complete view of Hall Probe, Undulator and Beampipe. 


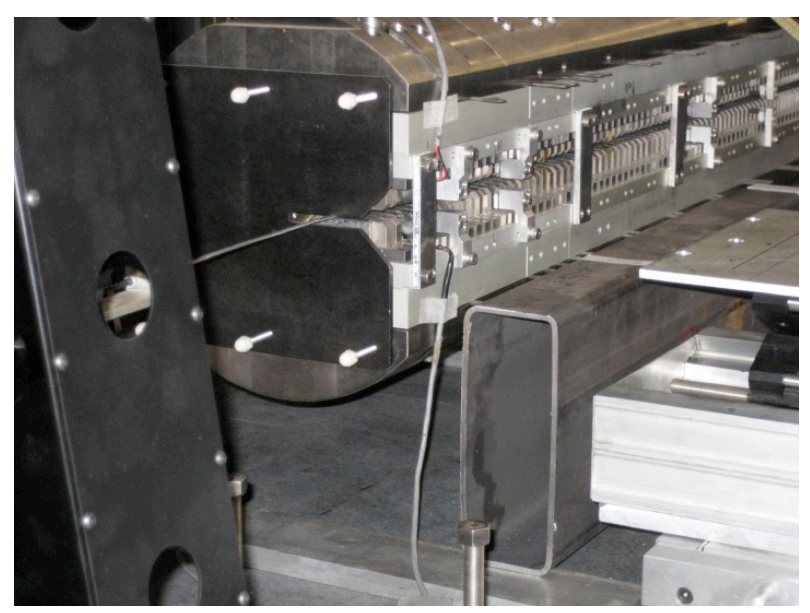

View of Steel Strongback

Each test was completed the same way. First a scan was conducted without the insertion of the beam pipe or shims. Matlab was then used to analyze the data and find the field peaks. These peaks were used for the analysis because in the areas between the field peaks, the field gradient in $\mathrm{Z}$ was too steep for comparison between runs due to small errors in position. After the first scan, a second scan with the exact same setup was completed. Using the difference in the peak fields between these scans, the error of the measurements was calculated, and used to set the error bars for the next comparison. A third scan was then done with the beampipe inserted and using the RMS difference from the first two scans as error bars. This was used to determine the shielding from the beampipe as well as provide a baseline with which to compare measurements with shims in place.

To measure the effect of the shims, the beampipe was then removed and the shims to be tested inserted into the magnet. The same measurement procedure was followed as before, using two identical scans to determine the RMS variation due to changes in the hall probe gain, and then a final scan was completed with the beampipe in place. By comparing the difference plots from the first set of scans with just a beampipe, to the second scan with a beampipe and shims, we were able to determine the changes in the shielding effect of the beampipe due to shim placement.

Using the same hall probe and stage setup as the beampipe measurements, a similar set of tests was conducted for the steel strongback. Two scans were done without the strongback in place, to determine the RMS variation of the measurements, then the strongback sample was placed in approximately the same position it would occupy in the tunnel. Using the same methods as above with and without the strongback instead of the beampipe, it's effect was determined.

In the following plot the peaks were located by performing a fit to the 5 points at and around the strongest field measured, and then using that fit to determine the position and strength of the actual peak field. This method was used due to the high field gradient found in the undulator. By selecting the peaks with a fit, small changes in the position of the actual measurements would not effect the data. The peaks were then used for the subsequent comparisons. Where the repeatability of our measurements was shown. 


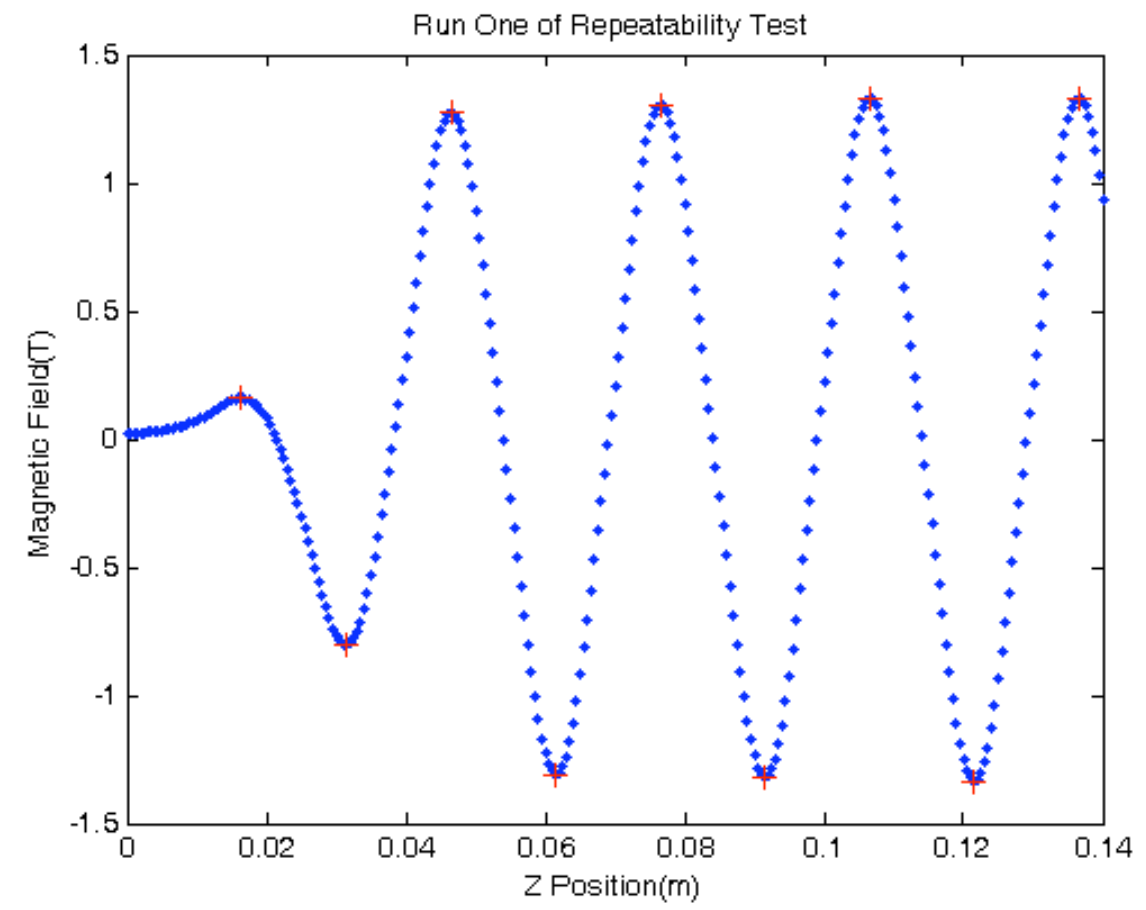

Scan of the undulator field. The red marks indicate the peak locations.

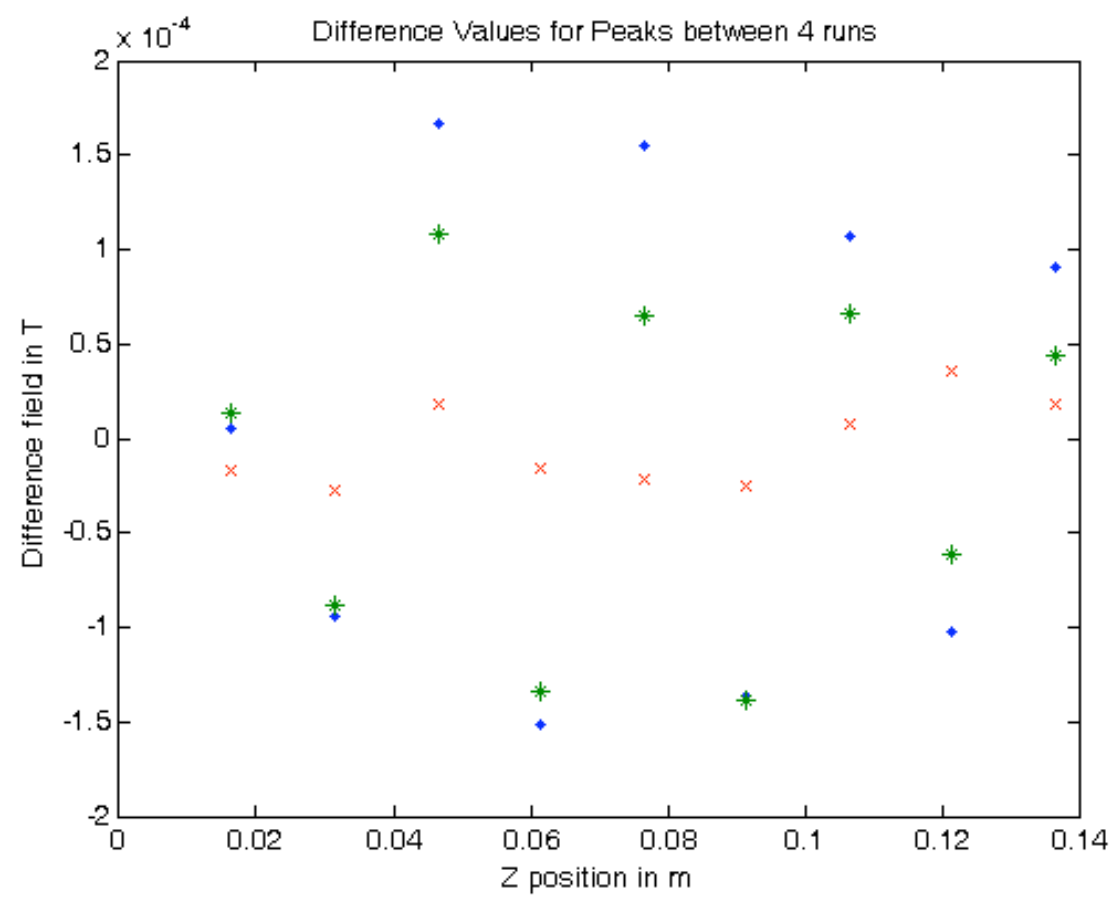

Summary plot of the peaks from four runs conducted in series. 


\section{Results}

\subsection{Beam Pipe Shielding}

The first set of measurements determined the overall shielding effect of the beampipe. Although there was some variation in the shielding effect from point to point on the scan, these variations are mostly within error bars, and the ones that are not can probably be attributed to fringe effects near to the edges of the beampipe. This plot shows the differnce in the field peaks between the undulator without the beampipe in place and with the beampipe in place.

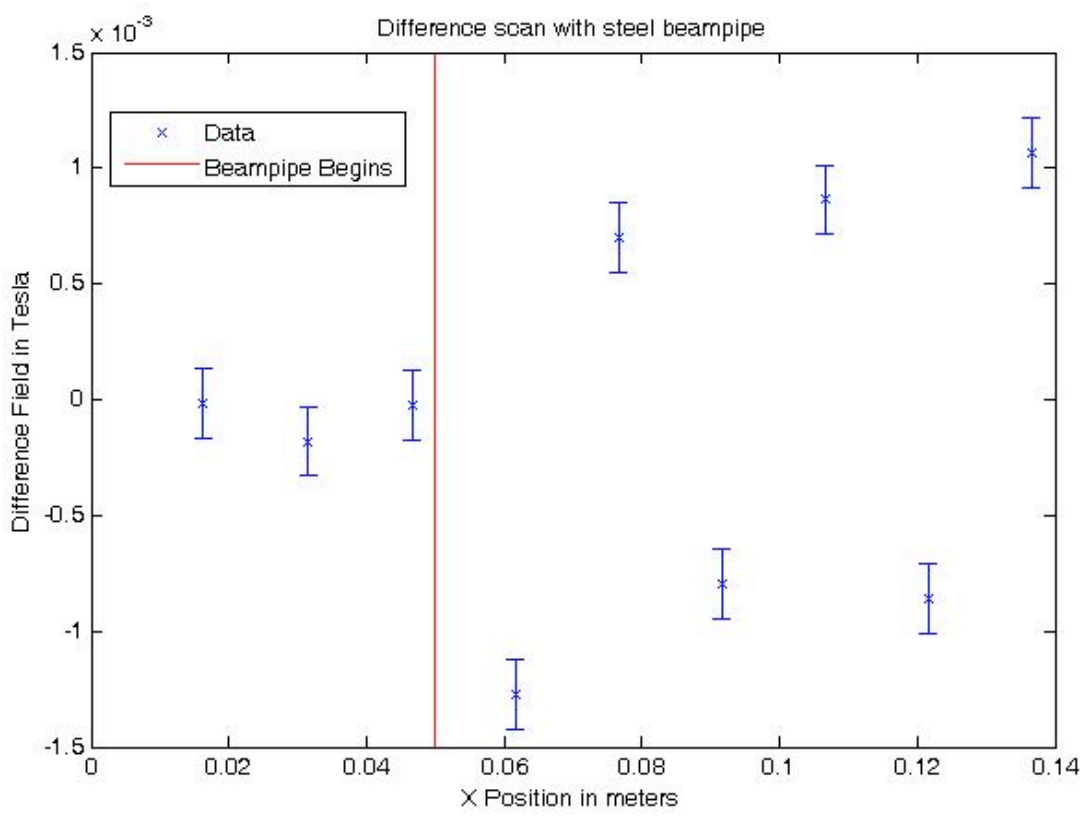

The average shielding effect of 8-12 gauss.

\subsection{Effect Due to Phase Shims}

The second set of measurements determined the change in the shielding effect of the beam pipe with set of phase shims placed in the undulator. The plot shows a dramatic 4 gauss increase when the $.3 \mathrm{~mm}$ phase shims were placed in the magnet. This plot shows three sets of scans, one which was just a comparison of beampipe and no beampipe, one which was a comparison of beampipe with no beampipe with the shims in place, and one which was a comparison of beampipe and no beampipe with the shims in place and the beampipe moved up against the shims to show that it was a purely magnetic effect changing the shielding and not a mechanical effect due to the phase shims pressing on the beampipe. 


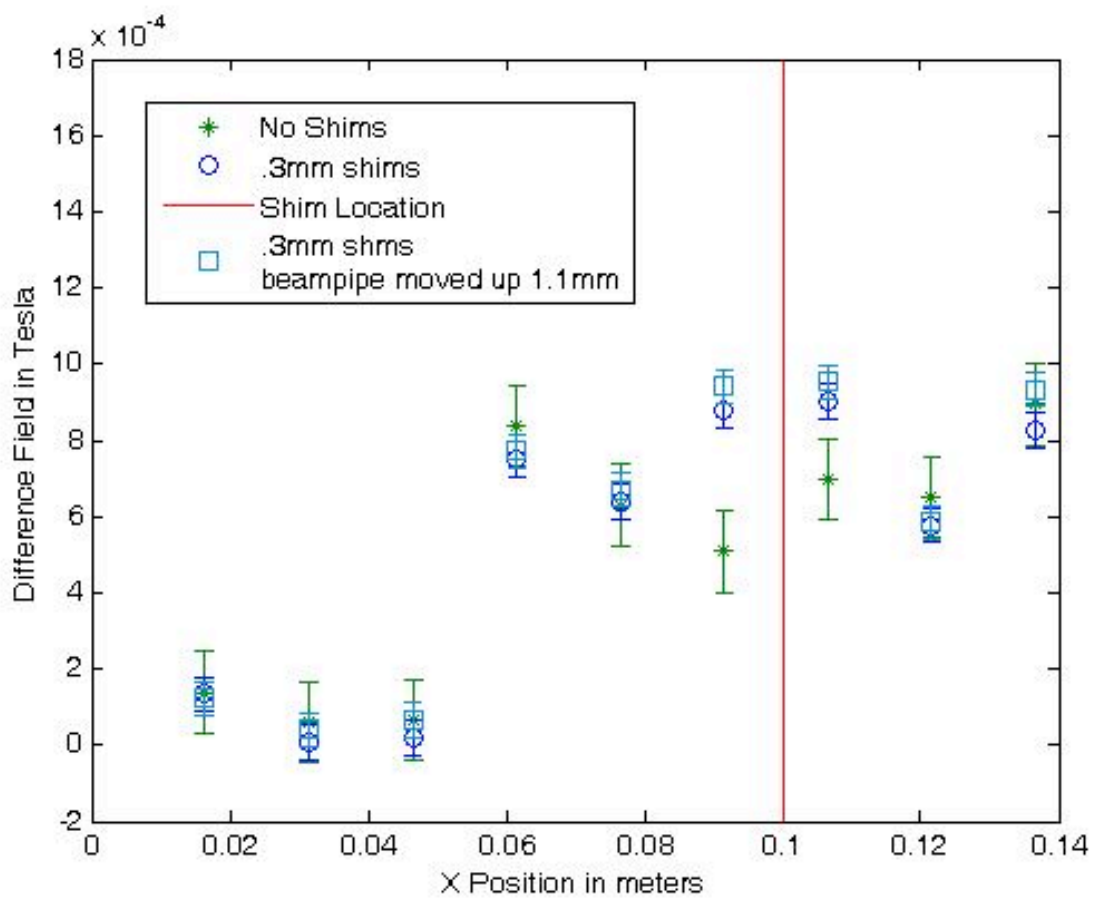

Plot of the difference scans with and without shims.

At the shim location, which was $10 \mathrm{~cm}$ inside the undulator, the shielding effect of the beam pipe was increased $\sim 4$ gauss due to the presense of the phase shims.

\subsection{Effect Due to Steel Strongback}

Although the anticipated effect due to the steel strongback was less than that found in the phase shims, it was still a significant effect. The data shows a clear effect which was probably due to a combination of the strongback being magnetized and the strongback's attenuating earths field. This plot is of the difference between the peaks with and without the steel strongback in place. 


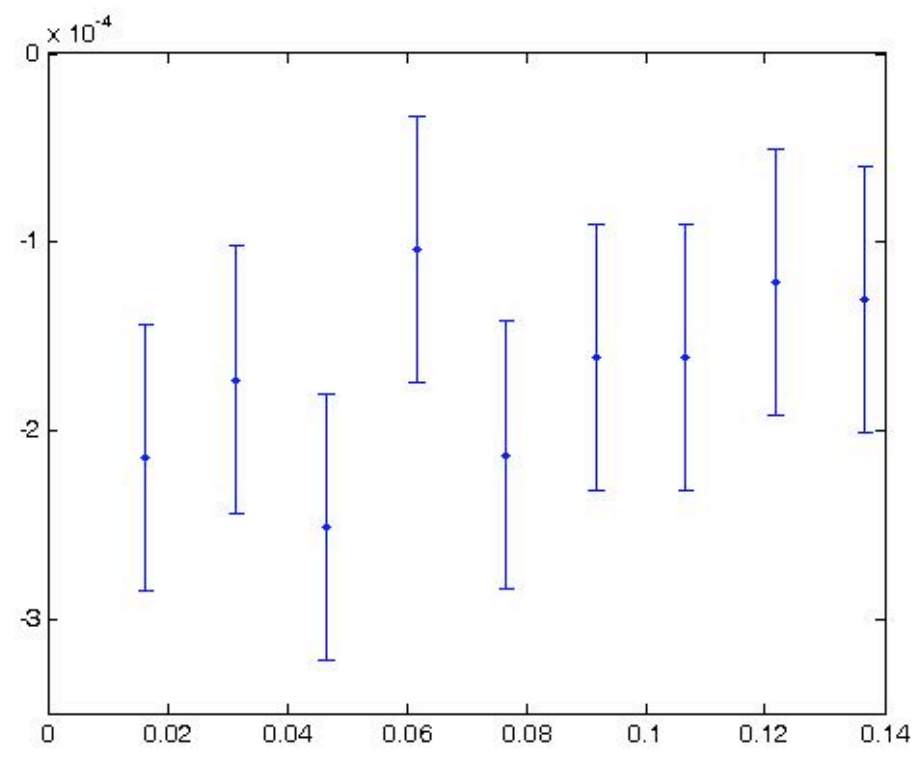

Plot of the peak difference in scans with and without the steel strongback.

As you can see, there is a dipole field $\sim 2$ guass introduced across the undulator. However, the effect was reduced by placing the mu metal shields, which are sections of mu metal installed to reduce the effect of Earth's field on the undulator.

\section{Conclusions}

Measuerments show that the beampipe introduced a net shielding effect of $\sim 10$ gauss. The shielding effect was modified by the placement of $.3 \mathrm{~mm}$ phase shims, on the order of 4 guass. This effect was considered to be large and problematic as the phase shims are placed in the magnet on an as-needed basis and have no specific pattern. This interaction of the shims with the steel beampipe led us to suggest that the beampipe be switched to aluminium instead of the originally proposed stainless steel. The measurements with the strongback in place showed a $\sim 2$ guass dipole magnetic field introduced across the undulator. The effect was greatly reduced by placing the mu metal on the undulator. 